\title{
Monte carlo simulations of the TOSCA spectrometer: Assessment of current performance and future upgrades
}

\author{
Roberto S. Pinna ${ }^{1,2}$, Svemir Rudić ${ }^{1}$, Stewart F. Parker ${ }^{1}$, Giuseppe Gorini ${ }^{2}$ and Felix Fernandez-Alonso ${ }^{1,3}$, a \\ ${ }^{1}$ ISIS Facility, Rutherford Appleton Laboratory, Chilton, Didcot OX11 0QX, UK \\ ${ }^{2}$ CNISM, Università degli Studi di Milano-Bicocca, Piazza della Scienza 3, 20126 Milano, Italy \\ ${ }^{3}$ Department of Physics and Astronomy, University College London, Gower Street, London WC1E 6BT, UK
}

\begin{abstract}
We describe and assess the performance of a detailed computational description of the high-resolution TOSCA spectrometer at ISIS using neutron-transport Monte Carlo simulations. Extensive calculations using the McStas software package have been performed using the present instrument geometry and compared with available experimental data. The agreement between expected and measured performance is satisfactory in terms of the incident flux spectrum, associated time structure, and spectroscopic resolution. Encouraged by these results, we also consider the upgrade of the primary spectrometer with a tapered high- $m$ guide. This instrument development offers the exciting prospects of providing order-of-magnitude gains in detected neutron flux over the energy-transfer range of the instrument whilst preserving its outstanding spectroscopic capabilities.
\end{abstract}

\section{Introduction}

TOSCA [1] is a broadband, indirect-geometry inelastic neutron spectrometer optimised for high-resolution chemical spectroscopy up to energy transfers of ca. $500(4000) \mathrm{meV}\left(\mathrm{cm}^{-1}\right)$ in neutron-energy loss $(1 \mathrm{meV}=$ $\left.8.065 \mathrm{~cm}^{-1}\right)$. The secondary spectrometer sits at $17 \mathrm{~m}$ from a room-temperature water moderator on ISIS Target Station I and has been operational for over a decade, superseding its predecessor TFXA [2].

As shown schematically in Fig. 1, energy analysis is performed after the interaction of the incident neutron beam with the sample under investigation. Scattered neutrons are Bragg-reflected from a pyrolytic graphite (PG) analyser and higher-order reflections beyond (002) are suppressed by a cooled Be filter $(\mathrm{T}<50 \mathrm{~K})$ so as to define a unique final energy of $\sim 4(32) \mathrm{meV}\left(\mathrm{cm}^{-1}\right)$. The instrument is comprised of a total of ten inelastic banks each having thirteen squashed ${ }^{3} \mathrm{He}$ tubes with an effective length(thickness) of $25(0.25) \mathrm{cm}$. Five banks are located in forward scattering (scattering angle $\sim 45^{\circ}$ ) and five in backscattering $\left(\sim 135^{\circ}\right)$. The use of a low (fixed) final energy translates into a direct relationship between energy transfer $\left(\mathrm{E}_{\mathrm{T}}, \mathrm{meV}\right)$ and momentum transfer $(\mathrm{Q}$, $\AA^{-1}$ ) such that $\mathrm{E}_{\mathrm{T}} \approx 2 \mathrm{Q}^{2}$. A disc chopper to prevent frame overlap is positioned at $8 \mathrm{~m}$ from the moderator. A recent chopper upgrade uses the 40-ms time frame during the operation of both target stations at ISIS to extend the incident-wavelength bandwidth of the instrument down to $-3(24) \mathrm{meV}\left(\mathrm{cm}^{-1}\right)$. At ISIS, this wide energy-transfer range provides overlap with both low (IRIS [3], LET [4], and OSIRIS [5]) and high energy-transfer spectrometers (MAPS [6] and VESUVIO [7]). TOSCA has been optimised to deliver an outstanding ('chemical') resolution

${ }^{a}$ Corresponding author: felix.fernandez-alonso@stfc.ac.uk across its spectral range as a consequence of several factors, including: a relatively narrow energy bandpass of the PG002/Be analyser system; tight moderator pulse widths; a long incident flight path; and a time- and energyfocused detector geometry. Instrument backgrounds are negligible, thus low-cross-section measurements beyond hydrogen-containing materials are not only feasible but also a growing area of research on the instrument. Quantitatively, the above design choices translate into an absolute spectral resolution of $\sim 0.3 \mathrm{meV}$ for elastic events $\left(\mathrm{E}_{\mathrm{T}}=0 \mathrm{meV}\right)$ and $\sim 12 \mathrm{meV}$ at $\mathrm{E}_{\mathrm{T}}=500 \mathrm{meV}$. The latter energy transfer lies in the vicinity of the $\mathrm{H}-\mathrm{H}$ stretch mode in molecular hydrogen and, therefore, represents an absolute upper bound for vibrational dynamics in condensed matter. TOSCA has also had modest highresolution diffraction capabilities from its inception, with two pairs of ${ }^{3} \mathrm{He}$ tubes located in backscattering geometry over the angular range $\pm 177-179^{\circ}$.

In terms of its science programme, TOSCA has set the standard for broadband chemical spectroscopy with neutrons over the past decade or so, as evidenced by a ratio of publication to accepted proposal of ca. 0.8 in the past five years - this figure increases to 0.9 if its vibrant Xpress access system is also taken into account [8]. It has also been the inspiration for next-generation chemical spectrometers such as VISION at the SNS, Oak Ridge, USA [9-11]. Owing to the higher source intensity of the SNS and the use of state-of-the-art neutron technology [11], VISION can provide significantly higher detected fluxes than TOSCA. The similarity of design also means that VISION should also enjoy negligible backgrounds. In addition, the new instrument IN1-Lagrange at ILL [12] delivers a much higher average flux than TOSCA (and possibly even more than VISION), as inferred from recent studies on this instrument by some of the authors $[13,14]$. However, the resolution of IN1-Lagrange 

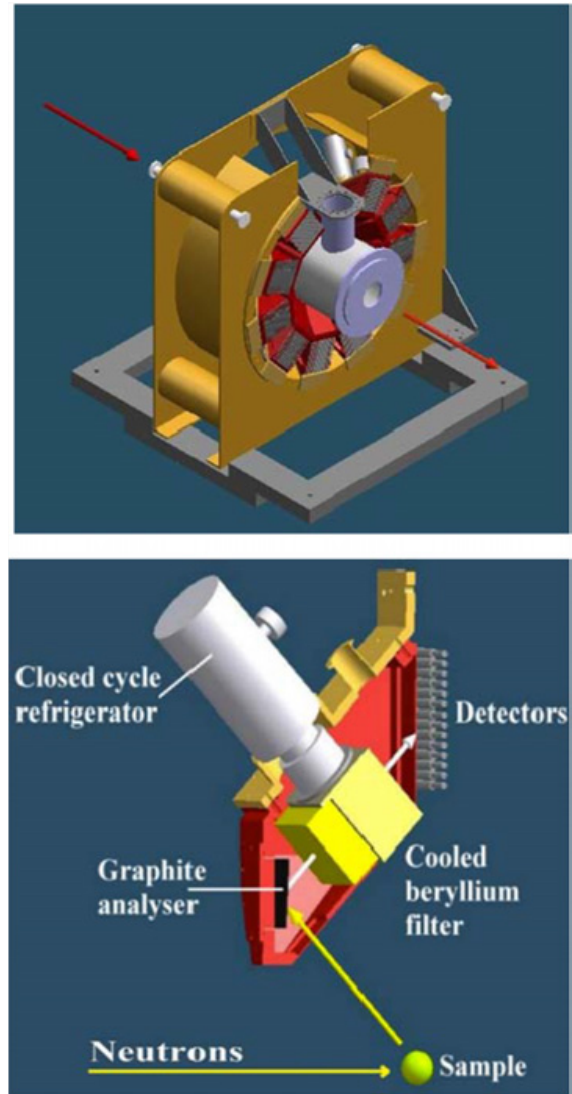

Figure 1. Top: schematic drawing of the TOSCA secondary spectrometer. The red arrows indicate the direction of incident (top left) and transmitted (bottom right) neutron beams. Bottom: detailed view of an inelastic bank. For further details, see the text. (Credit: Consiglio Nazionale delle Ricerche, Italy.)

remains comparable to TFXA (i.e., similar to that of most currently operating instruments). As a neutron spectrometer at a steady reactor source, background runs will always be important, necessarily restricting signal-tobackground ratios to moderate values. It is also a scanning spectrometer and spectral contamination by higher orders from the incident monochromator may be problematic to discern subtle spectral features, particularly at low energy transfers. Nonetheless, IN1-Lagrange is probably the spectrometer of choice for very small samples (few mg or less) and extensive parametric studies, particularly over narrow energy-transfer ranges.

As a world-leading neutron spectrometer, TOSCA has been constantly evolving, with significant improvements to its performance over the past few years as a result of a number of small and medium upgrades [15]. In this work, we describe parallel efforts to provide (for the first time) an accurate computational description of the instrument using neutron-transport Monte Carlo simulations, with a view on a detailed assessment of potential upgrade routes in the short, medium, and long terms.

\section{Current performance}

All Monte Carlo simulations have been performed using the parallelised version of the McStas software package [16]. Computations were performed on the SCARF-RAL

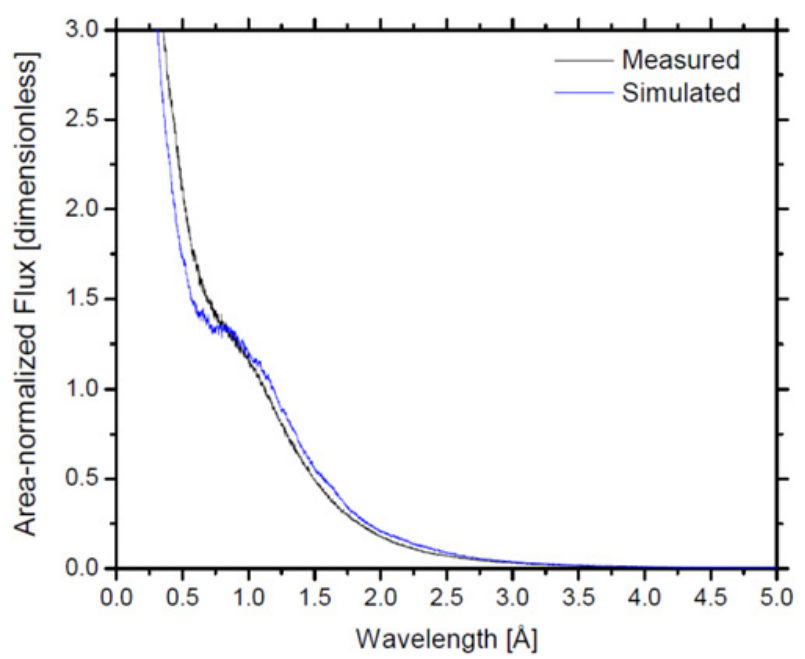

Figure 2. Measured and simulated incident neutron spectra on TOSCA. To facilitate comparison, both histograms have been normalised over the neutron-wavelength range $0.3-5.0 \AA$. For further details, see the text.

cluster [17] using up to 128 nodes. The geometry of both primary and secondary spectrometers has been taken from current engineering drawings of the instrument.

Figure 2 shows a comparison between experimental and simulated incident neutron spectra at the position of the primary monitor. This monitor is located upstream from the sample position at $15.795 \mathrm{~m}$ from the roomtemperature water moderator. Experimental raw data have been corrected by the (measured) wavelength-dependent efficiency of this lithium-glass scintillator detector over the incident-wavelength range of the instrument $(0.3-5.0 \AA)$. Simulated spectra were calculated using the ISIS Target Station I water-moderator module released in 2008 and available on the McStas package.

Overall, the Monte Carlo calculations provide a satisfactory description of the experimental incident spectrum, although some differences are to be noted in terms of the extent of the "moderated hump," which appears to be more pronounced in the simulated dataset. These differences are of the order of $20-25 \%$ around the fingerprint region of vibrational spectra (10$125 \mathrm{meV}$ or $100-1000 \mathrm{~cm}^{-1}$ ). Notwithstanding the above differences, both experimental and simulated data evince a predominantly epithermal-looking incident spectrum. We also note that these discrepancies have a minor effect on reduced inelastic neutron spectra, as these are always normalised to the incident neutron-flux distribution prior to subsequent analysis or comparison with the predictions of computational models.

In addition to incident neutron spectra, the performance of an inverted-geometry instrument like TOSCA critically depends on the time structure of neutron pulses reaching the sample position. On TOSCA, experimental access to this information is facilitated by the availability of a high-resolution diffraction bank in backscattering geometry. In this configuration, the observed time widths of well-defined Bragg reflections become most sensitive to the temporal spread of neutron pulses arriving at the sample position, as detailed in recent Monte Carlo 


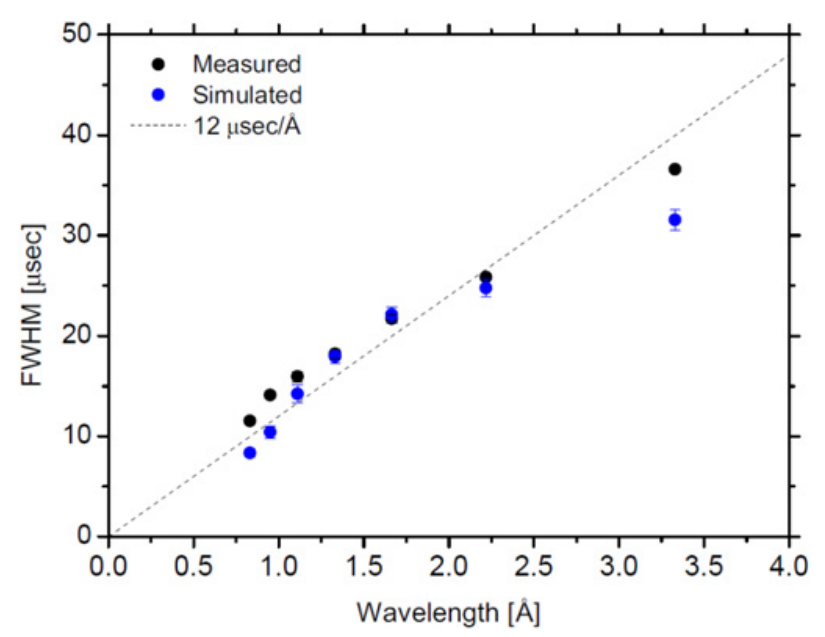

Figure 3. Measured and simulated time widths as a function of incident neutron wavelength. For further details, see the text.

simulations of the OSIRIS spectrometer [18]. In the particular case of TOSCA, measurements at a temperature of $10 \mathrm{~K}$ were performed on a $40 \times 20 \times 2 \mathrm{~mm}^{3}$ highly oriented pyrolytic graphite (HOPG) standard with a mosaicity of $0.8 \pm 0.2^{\circ}$ along the $c$-axis. This standard was aligned with the $a-b$ plane perpendicular to the incident beam and its $c$-axis rotated so as to maximise (001) Bragg intensities on detector 147 , located at a distance of $1.21 \mathrm{~m}$ upstream from the sample position at a scattering angle of $178.28^{\circ}$. For comparison, an identical experimental setup was implemented on McStas.

Figure 3 shows a comparison of the wavelength dependence of time widths associated with (00l) HOPG Bragg reflections, as obtained from least-squares fits of both experimental and simulated data using a type-I extreme-value line shape. All widths reported in this work correspond to full-width-at-half-maxima (FWHM) and the associated error bars denote uncertainties in the data fits. In both cases, the widths are dominated by the temporal response of the primary spectrometer and, in particular, the time structure emerging from the moderator face. Other contributions associated with time uncertainties between moderator and sample are relatively minor in comparison. Overall, the agreement between experiment and simulation is quite satisfactory, particularly in terms of an overall increase in FWHM with neutron wavelength in the thermal and cold regimes. At the longest wavelength investigated $(3.33 \AA$ ), simulations are within $10-15 \%$ of experimental values, and consistently provide a safe lower bound to observation. These results are also in agreement with a moderator term of $\sim 12 \mu \mathrm{s} / \AA$ inferred from previous calibrations of the instrument $[2,19]$. On the basis of the present comparison, this moderator term provides a good description of time structure below ca. 3.0 А. At the higher wavelength investigated, the time width shows signs of saturation, as one would expect for the moderation of cold neutrons at a short-pulse spallation source [20]. These results also highlight the superb diffraction capabilities of the instrument, characterised by a $d$-spacing resolution of $\Delta d / d \sim 5 \times 10^{-3}$ over its operating wavelength range. This unique feature of inverted-geometry instrumentation

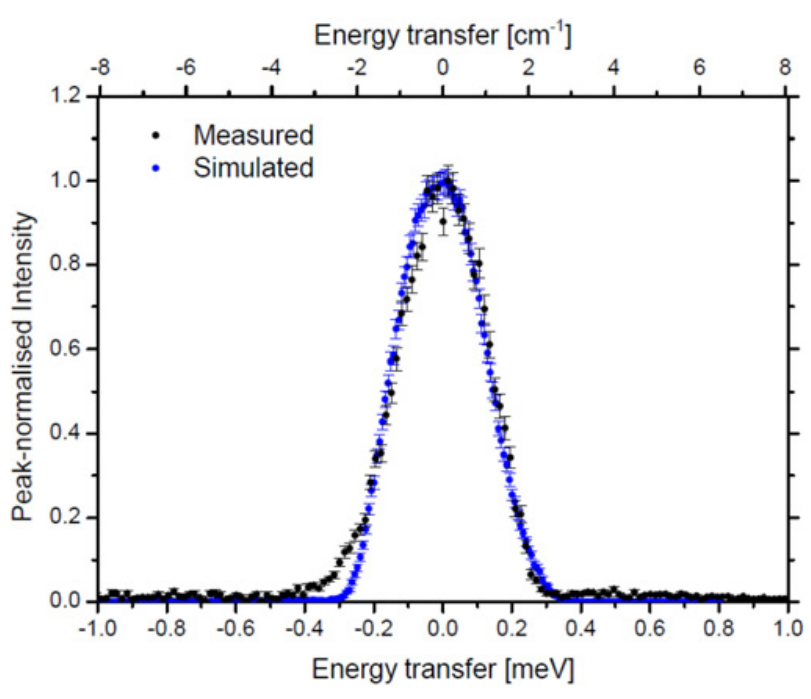

Figure 4. Experimental and simulated spectra of ice at a temperature of $10 \mathrm{~K}$ using the inelastic backscattering bank at a (nominal) scattering angle of $135^{\circ}$.

at a short-pulse spallation neutron source could be further exploited via a significant increase in detector area relative to current (and quite modest) capabilities on TOSCA, as already demonstrated on the low-energy spectrometer OSIRIS [5].

The above comparisons between experimental and simulated performance were primarily concerned with a characterisation of the primary spectrometer. To assess the validity of our current description of the entire instrument, we have also compared experimental and simulated spectra around the elastic line for ice at a temperature of $10 \mathrm{~K}$, as shown in Fig. 4. For the purposes of benchmarking the McStas simulations, this case represents a convenient scenario characterised by high scattering levels (potentially leading to an increase in instrumental backgrounds), as well as comparable contributions to the resolution function from both primary (moderator) and secondary spectrometers (inelastic banks). As shown in Fig. 4, the agreement between experiment (FWHM = $0.3 \mathrm{meV})$ and simulation $(0.29 \mathrm{meV})$ is excellent aside from a slight excess in scattered intensity for neutronenergy gain processes. Elucidating the precise origin of this second-order feature in the observed spectrum is beyond the scope of the present work, as it would require a detailed and systematic line shape analysis similar to that conducted recently for the OSIRIS spectrometer [18]. Overall, these results further confirm the adequacy of our computational model for a quantitative description of the spectroscopic response of the instrument in its present incarnation.

\section{More neutrons for chemistry}

In view of current developments in chemical spectroscopy with neutrons around the globe, the current sensitivity of TOSCA could be greatly enhanced via the provision of a neutron guide in the primary spectrometer. To assess possible gain factors relative to current capabilities, extensive McStas simulations have been performed for a 


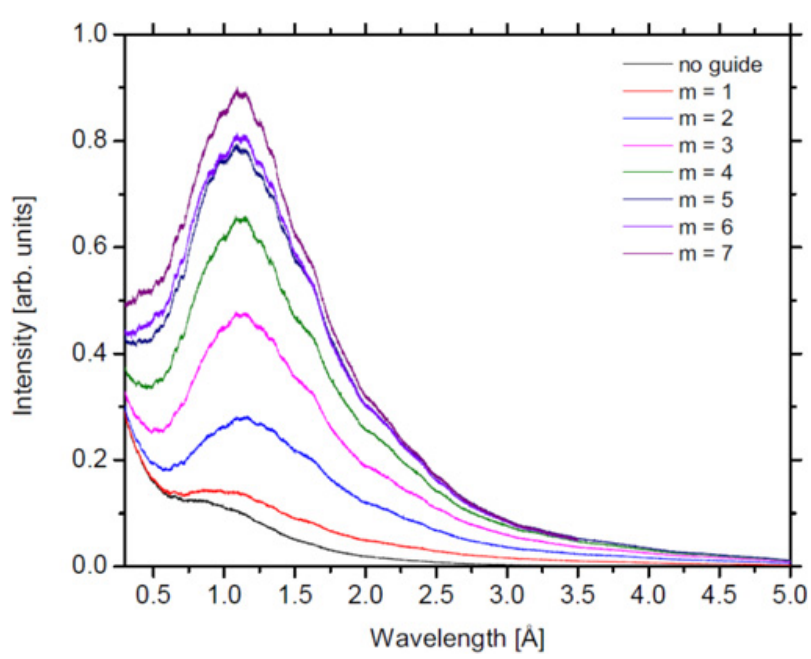

Figure 5. Incident-wavelength spectra at the sample position as a function of guide $m$-number. The black trace corresponds to the current spectrometer (no guide).

range of guide configurations using realistic reflectivity profiles as described in Chapter 5 of the McStas user manual [16]. This exercise must necessarily take into account the cost effectiveness of any proposed guide geometry, as well as other spatial and operational constraints on a busy instrument like TOSCA.

A tapered guide represents the most sensible geometry to transmit neutrons over a wide wavelength range. Such a guide can be placed at a minimum distance from the moderator of $1.625 \mathrm{~m}$ with a cross sectional area of $10 \times$ $10 \mathrm{~cm}^{2}$, followed by thirteen independent sections ending at a distance of $0.75 \mathrm{~m}$ from the sample position $(4 \times$ $4 \mathrm{~cm}^{2}$ ). Figure 5 shows how the flux at the sample position would evolve as a function of guide $m$-number.

The highest absolute gains are observed around $1 \AA$, approaching an order-of-magnitude enhancement in flux for the highest $m$-numbers investigated. We also note a monotonic (and quite significant) increase in flux up to $m \sim 5-6$, values which are well within reach owing to advances in neutron-guide technology over the past decade. In relative terms, the largest gains are observed at the longest wavelengths, with factors exceeding 50 around the elastic line of the instrument at ca. $5 \AA$. The energy transfers accessible in this neutron-wavelength range correspond to the hard-to-access $\mathrm{THz}$ range in optical spectroscopy $\left(1 \mathrm{THz}=4.13 \mathrm{meV}=33.3 \mathrm{~cm}^{-1}\right)$, as well as provide much-needed overlap with the higherresolution instruments IRIS and OSIRIS at ISIS. These two instruments have already demonstrated a phenomenal energy resolution for inelastic studies up to energy transfers of ca. 20(160) $\mathrm{meV}\left(\mathrm{cm}^{-1}\right)$ [21-23], and could very well complement the broadband capabilities afforded by TOSCA at shorter wavelengths. The above flux gains in the $\mathrm{THz}$ window drop relatively quickly with decreasing wavelength to values of 2-3 below $1 \AA$. We also find that a progressive increase in $m$-number across the primary spectrometer tends to provide a more balanced gain across the spectral range of the instrument. Likewise, the net transport of high incident wavelengths (4-5 $\AA$ ) can be maximised by having a guide insert inside the shutter

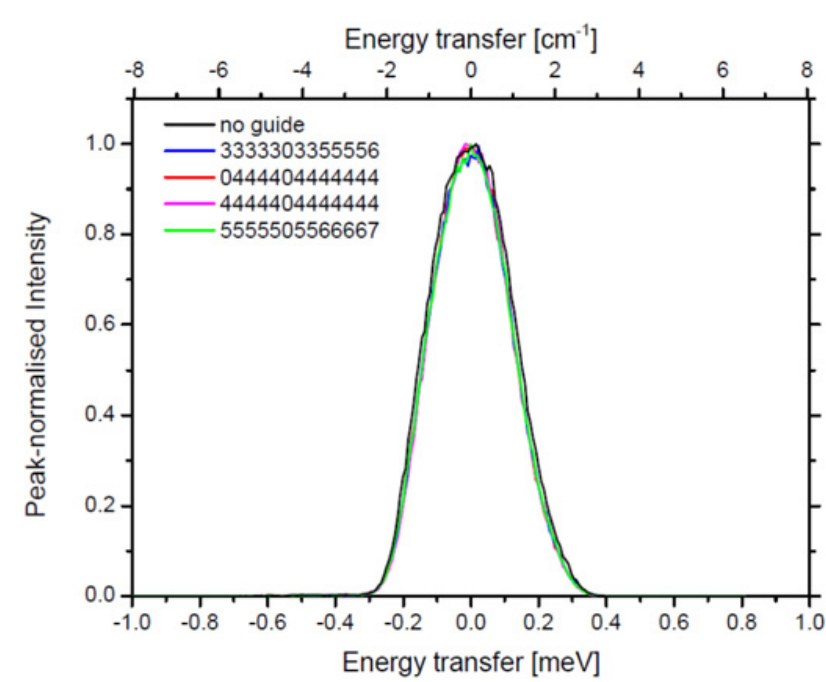

Figure 6. Simulated spectra of ice at a temperature of $10 \mathrm{~K}$ using the inelastic backscattering bank at $135^{\circ}$. The legend indicates different guide configurations as described in the text.

assembly, as close to the moderator face as present space constraints on ISIS Target Station I may allow. A high $m$-number closer to the sample can also increase the flux at the sample by factors of 2-3 for the shorter wavelengths ca. $1 \AA$.

From extensive simulations to assess the relative performance of a total of forty different guide geometries, the configuration of choice corresponds to [5555505566667], where each single digit within square brackets denotes the $m$-value for each independent section along the primary spectrometer, starting closest to the moderator face. Predicted gains for this configuration are 52 and 3 for the highest and lowest incident wavelengths available on the instrument, respectively. Use of a high- $m$ guide $(m>4)$ close to the source ensures reasonable gain factors above 20(160) $\mathrm{meV}\left(\mathrm{cm}^{-1}\right)$.

As an additional test, Fig. 6 shows that the spectral resolution of the instrument around the elastic line is largely insensitive to a rather substantial increase in the $m$-number of the guide in the primary spectrometer. These results are to be taken as a worst-case scenario, given the linear dependence of beam divergence on both incident wavelength and $m$-number. We therefore conclude that the predicted gains reported in Fig. 5 are not accompanied by a concomitant degradation of the spectroscopic capabilities presently afforded by TOSCA.

\section{Outlook}

Monte-Carlo simulations of the TOSCA spectrometer using the McStas software package provide a satisfactory description of the current performance of the instrument in terms of incident-flux spectra, associated time structure, and spectroscopic response. Encouraged by these results, we have also assessed potential flux gains associated with the installation of a neutron guide in the primary spectrometer. Unlike a decade ago when the instrument became operational, judicious use of state-of-the-art guide technology to upgrade the primary spectrometer offers 
the exciting prospects of providing order-of-magnitude gains in sensitivity whilst preserving the outstanding spectroscopic capabilities of the instrument.

On the scientific front, this much-needed upgrade implies a concomitant reduction in counting times, a transformational development as it would enable detailed studies of industrially relevant systems exhibiting low scattering cross sections. To name a few, these include studies of molecular adsorbates such as $\mathrm{CO}$, NO, or $\mathrm{SO}_{2}$ on catalysts, where inelastic neutron scattering can uniquely provide information on the low-energy modes underpinning adsorbate-surface interactions; or of proton-conducting pathways in charge-storage materials containing minute amounts of hydrogen. Detailed parametric studies would become routine as well, particularly for hydrogen-containing systems such as polymers and nanostructured materials. Moreover, the (very popular) TOSCA Xpress service could also be expanded and automated beyond its current remit to provide an efficient outreach tool.

In conjunction with ongoing efforts at ISIS to improve neutronic performance, the upgrade possibilities described herein will certainly keep TOSCA at the forefront of chemical spectroscopy with neutrons in the foreseeable future.

The authors gratefully acknowledge the UK Science \& Technology Facilities Council for financial support, access to beam time at ISIS, and use of the e-Science SCARF cluster at the Rutherford Appleton Laboratory. We also thank Peter Philips and Colin French from the ISIS Experimental Operations Division for the precise machining of the HOPG standards. This work has been partially supported within the framework of past and present CNR-STFC agreements for collaborative research between Italy and ISIS.

\section{References}

[1] www.isis.stfc.ac.uk/instruments/tosca/ (last accessed 22 September 2014)

[2] D. Colognesi, M. Celli, F. Cilloco, R. J. Newport, S.F. Parker, V. Rossi-Albertini, F. Sacchetti, J. Tomkinson, and M. Zoppi, Appl. Phys. A 74, S64S66 (2002)

[3] www.isis.stfc.ac.uk/instruments/iris/ (last accessed 22 September 2014)
[4] www.isis.stfc.ac.uk/instruments/let/ (last accessed 22 September 2014)

[5] www.isis.stfc.ac.uk/instruments/osiris/ (last accessed 22 September 2014)

[6] www.isis.stfc.ac.uk/instruments/maps/ (last accessed 22 September 2014)

[7] www.isis.stfc.ac.uk/instruments/vesuvio/ (last accessed 22 September 2014)

[8] www.isis.stfc.ac.uk/apply-for-beamtime/ (last accessed 22 September 2014)

[9] P.A. Seeger, L.L. Daemen, and J.Z. Larese, Nucl. Instr. Meth. Phys. Res. A 604, 709 (2009)

[10] neutrons.ornl.gov/vision/ (last accessed 22 September 2014)

[11] C. Wildgruber, personal communication

[12] www.ill.eu/instruments-support/instrumen ts-groups/instruments/in1-taslagrange/ (last accessed 22 September 2014)

[13] F. Fernandez-Alonso, M.J. Gutmann, S. Mukhopadhyay, D.B. Jochym, K. Refson, M. Jura, M. Krzystyniak, M. Jimenez-Ruiz, and A. Wagner, J. Phys. Soc. Jpn. 82, SA001 (2013)

[14] S. Mukhopadhyay, M.J. Gutmann, M. Jura, D.B. Jochym, M. Jimenez-Ruiz, S. Sturniolo, K. Refson, and F. Fernandez-Alonso, Chem. Phys. 427, 95 (2013)

[15] S.F. Parker et al., J. Phys.: Conf. Series (in the press, 2014)

[16] www.mcstas.org/ (last accessed 22 September 2014)

[17] www.scarf.rl.ac.uk/ (last accessed 22 September 2014)

[18] K. Pokhilchuk, Rutherford Appleton Laboratory Technical Report RAL-TR-2013-008 (Chilton, 2013, ISSN 1358-6254). Report available at: epubs.stfc.ac.uk/work/65985 (last accessed 22 September 2014)

[19] V. Rossi Albertini, D. Colognesi, and J. Tomkinson, J. Neutron Research 8, 245 (2000)

[20] M. Arai, in Neutron Scattering - Fundamentals, F. Fernandez-Alonso and D.L. Price eds. (Academic Press, New York, 2013), Chapter 3

[21] F. Fernandez-Alonso et al., Phys. Rev. Lett. 98, 215503 (2007)

[22] A. Lovell et al., Phys. Rev. Lett. 101, 126101 (2008)

[23] F. Demmel et al., Eur. Phys. J. (accepted, 2014) 